\title{
Correction to: Yield curve shapes of Vasicek interest rate models, measure transformations and an application for the simulation of pension products
}

\author{
Franziska Diez ${ }^{1} \cdot$ Ralf Korn $^{1,2}$ (1)
}

Published online: 18 May 2021

(c) EAJ Association 2021

\section{Correction to: European Actuarial Journal (2020) 10:91-120 https://doi.org/10.1007/s13385-019-00214-0}

Due to an error in the proof of Lemma 1 in the appendix of the original article, the proof of the assertions (c) and (d) of Theorem 4 on the existence of humped and dipped yield curves in the two-factor Vasicek model needs a modification. However, the assertions stay valid. It can directly be realized that the proof of Lemma 1 holds for $h:=f-g$ and not for $h:=f+g$ as we falsely claimed. Consequently, Lemma 1 can not be applied. Using the notation of Section 3 in the original article, we will reformulate Theorem 4 slightly and then give the corrected proof for humped and dipped yield curves.

Theorem 4 In a two-factor Vasicek model defined as in Eq. (13), the following assertions hold under the assumption of $a \neq b$ :
(a) There are normal yield curves but only for $r(t)<y_{\text {asymp }}$,
(b) there are inverse yield curves but only for $r(t)>y_{\text {asymp }}$,
(c) there are humped yield curves for $r(t) \geq y_{\text {asymp }}$,
(d) there are dipped yield curves for $r(t) \leq y_{\text {asymp }}$.

Before we prove the theorem, we introduce two useful lemmas.

Lemma 1 Let $D \subseteq \mathbb{R}$ be a convex set. Let $f, g: D \rightarrow \mathbb{R}$ be strictly concave (convex) functions and $f(x) \neq g(x)$. Then, both functions have a maximum of two intersections.

The original article can be found online at https://doi.org/10.1007/s13385-019-00214-0.

Ralf Korn

korn@mathematik.uni.kl.de

Franziska Diez

Franziska.Diez@itwm.fraunhofer.de

1 Department of Financial Mathematics, Fraunhofer ITWM, 67663 Kaiserslautern, Germany

2 Department of Mathematics, University of Kaiserslautern, 67653 Kaiserslautern, Germany 
Proof We prove this lemma for $f$ and $g$ being strictly concave. For $f$ and $g$ being strictly convex, the proof follows analogously.

We assume that $f$ and $g$ have (at least) three intersections $x_{1}<x_{2}<x_{3}$. As $f$ and $g$ are strictly concave, for $\alpha \in(0 ; 1)$ we have

$$
\begin{aligned}
& f\left(\alpha x_{1}+(1-\alpha) x_{3}\right)>\alpha f\left(x_{1}\right)+(1-\alpha) f\left(x_{3}\right), \\
& g\left(\alpha x_{1}+(1-\alpha) x_{3}\right)>\alpha g\left(x_{1}\right)+(1-\alpha) g\left(x_{3}\right) .
\end{aligned}
$$

Taking the difference between the two inequalities, we obtain for $\alpha \in(0 ; 1)$

$$
f\left(\alpha x_{1}+(1-\alpha) x_{3}\right)-g\left(\alpha x_{1}+(1-\alpha) x_{3}\right)>0
$$

because $f\left(x_{1}\right)=g\left(x_{1}\right)$ and $f\left(x_{3}\right)=g\left(x_{3}\right)$. As $x_{1}<x_{2}<x_{3}$, there is an $\bar{\alpha} \in(0 ; 1)$ so that $x_{2}=\bar{\alpha} x_{1}+(1-\bar{\alpha}) x_{3}$ and the previous inequality holds for $x_{2}$

$$
f\left(x_{2}\right)-g\left(x_{2}\right)=f\left(\bar{\alpha} x_{1}+(1-\bar{\alpha}) x_{3}\right)-g\left(\bar{\alpha} x_{1}+(1-\bar{\alpha}) x_{3}\right)>0,
$$

which contradicts the assumption of $f\left(x_{2}\right)=g\left(x_{2}\right)$. Consequently, $f$ and $g$ can have at most two intersections.

Lemma 2 Let $a, \sigma, x>0$ and $K_{c}(x)$ be defined as

$$
K_{c}(x):=-\frac{\sigma^{2}}{4 a^{2}} \frac{B_{a}(x)}{x}\left(c-\left(1-e^{-a x}\right)\right) .
$$

The first derivative of $K_{c}(x)$ with respect to $x$ is

- strictly convex for $c \geq 1$,

- strictly concave for $c \leq-14$.

Proof First, we will prove that $\frac{\partial K_{c}(x)}{\partial x}$ is strictly convex for $c \geq 1$. The third derivative of $K_{c}(x)$ with respect to $x$ is

$$
\begin{gathered}
\frac{\partial^{3} K_{c}(x)}{\partial x^{3}}=-\frac{\sigma^{2}}{4 a^{2}}\left(\frac{\partial^{3} B_{a}(x) / x}{\partial x^{3}}\left(c-\left(1-e^{-a x}\right)\right)-3 a \frac{\partial^{2} B_{a}(x) / x}{\partial x^{2}} e^{-a x}\right. \\
\left.+3 a^{2} \frac{\partial^{B_{a}(x) / x}}{\partial x} e^{-a x}-a^{3} \frac{B_{a}(x)}{x} e^{-a x}\right) .
\end{gathered}
$$

Inspection of $\frac{B_{a}(x)}{x}$ delivers the following properties for $a, x>0$ :

$$
\begin{aligned}
& \frac{B_{a}(x)}{x}=\frac{1-e^{-a x}}{a x}>0, \frac{\partial^{B_{a}(x)} / x}{\partial x}=\frac{(1+a x) e^{-a x}-1}{a x^{2}}<0, \\
& \frac{\partial^{2} B_{a}(x) / x}{\partial x^{2}}=\frac{2\left(1-(1+a x) e^{-a x}\right)-a^{2} x^{2} e^{-a x}}{a x^{3}}>0, \\
& \frac{\partial^{3} B_{a}(x) / x}{\partial x^{3}}=\frac{6\left((1+a x) e^{-a x}-1\right)+3 a^{2} x^{2} e^{-a x}+a^{3} x^{3} e^{-a x}}{a x^{4}}<0 .
\end{aligned}
$$

These inequalities directly imply 


$$
\frac{\partial^{3} K_{c}(x)}{\partial x^{3}}>0
$$

for $c \geq 1$. Therefore, the first derivative of $K_{c}(x)$ is strictly convex for $c \geq 1$.

Next, we will prove that $\frac{\partial K_{c}(x)}{\partial x}$ is strictly concave for $c \leq-14$. The third derivative of $K_{c}(x)$ can be rewritten as

$$
\begin{aligned}
\frac{\partial^{3} K_{c}(x)}{\partial x^{3}}= & \frac{\sigma^{2}}{4 a^{2}}\left(\frac{6(c-1)+(2-c) e^{-a x}\left(a^{3} x^{3}+3 a^{2} x^{2}+6 a x+6\right)}{a x^{4}}\right. \\
& \left.-\frac{e^{-2 a x}\left(8 a^{3} x^{3}+12 a^{2} x^{2}+12 a x+6\right)}{a x^{4}}\right) \\
= & : \frac{\sigma^{2}}{4 a^{2}} \frac{6(c-1)+(2-c) z(x)-z(2 x)}{a x^{4}}=: \frac{\sigma^{2}}{4 a^{2}} \frac{k_{c}(x)}{a x^{4}} .
\end{aligned}
$$

Hence, $\frac{\partial^{3} K_{c}(x)}{\partial x^{3}}$ has the same sign as $k_{c}(x)$. Therefore, we consider $k_{c}(x)$. It attains the following limits

$$
\lim _{x \downarrow 0} k_{c}(x)=0, \quad \lim _{x \uparrow \infty} k_{c}(x)=6(c-1)
$$

and has the first derivative of

$$
\frac{\partial k_{c}(x)}{\partial x}=a^{4} x^{3} e^{-a x}\left(16 e^{-a x}-(2-c)\right) .
$$

As $a^{4} x^{3} e^{-a x}>0$ as well as $e^{-a x}$ is strictly monotonically decreasing from 1 to 0 for $a, x>0, \frac{\partial k_{c}(x)}{\partial x}$ has at most one zero in $x>0$ depending on $c$ and it is given by

$$
\bar{x}_{c}=-\frac{1}{a} \ln \left(\frac{2-c}{16}\right) .
$$

$\frac{\partial k_{c}(x)}{\partial x}$ does not have any zero in $x>0$ for $c \leq-14$ and we have

$$
\frac{\partial k_{c}(x)}{\partial x} \leq 16 a^{4} x^{3} e^{-a x}\left(e^{-a x}-1\right)<0 .
$$

Consequently ${ }_{3_{3} k_{c}}(x)$ is negative since it strictly monotonically decreases from zero on and thus $\frac{\partial^{3} K_{c}^{c}(x)}{\partial x^{3}}$ is negative for $x>0$. Therefore, the first derivative of $K_{c}(x)$ is strictly concave if $c \leq-14$.

We are now ready to give the corrected proof of the assertions (c) and (d) in Theorem 4:

Proof of Theorem 4 (c) We consider a short rate $r(t) \geq y_{\text {asymp }}$ and Transformation 1 


$$
\begin{aligned}
y(t, x)= & -\frac{\sigma^{2}}{4 a^{2}} \frac{B_{a}(x)}{x}\left(c_{A}-\left(1-e^{-a x}\right)\right)-\frac{\eta^{2}}{4 b^{2}} \frac{B_{b}(x)}{x}\left(c_{B}-\left(1-e^{-b x}\right)\right) \\
& \quad-\rho \frac{\sigma \eta}{a b} \frac{B_{a+b}(x)}{x}+\theta-\frac{\sigma^{2}}{2 a^{2}}-\frac{\eta^{2}}{2 b^{2}}-\rho \frac{\sigma \eta}{a b} \\
= & : K_{c_{A}}(x)+K_{c_{B}}(x)+R_{1}(x)+y_{\text {asymp }}
\end{aligned}
$$

where

$$
\begin{aligned}
& c_{A}:=-\frac{4 a^{2}}{\sigma^{2}}\left(X(t)+\psi(t)-\theta+\frac{\sigma^{2}}{2 a^{2}}+\rho \frac{\sigma \eta}{a b}\right), \\
& c_{B}:=-\frac{4 b^{2}}{\eta^{2}}\left(Y(t)+\frac{\eta^{2}}{2 b^{2}}+\rho \frac{\sigma \eta}{a b}\right) .
\end{aligned}
$$

We start with the case $a<b$. We choose $c_{B}$ as

$$
c_{B}>\max \left\{1 ;-\frac{4 b^{2}}{\eta^{2}}\left(\frac{a}{a-b}(r(t)-\theta)+\frac{\eta^{2}}{2 b^{2}}+\rho \frac{\sigma \eta}{a b}\right)\right\}
$$

and $c_{A}$ for $\rho \leq 0$ as

$$
c_{A} \leq \min \left\{-14 ;-\frac{a^{2} \eta^{2}}{b^{2} \sigma^{2}} c_{B}\right\}
$$

and for $\rho>0$ as

$$
c_{A} \leq \min \left\{-14 ;-\frac{4 a^{2}}{\sigma^{2}}\left(\frac{\eta^{2}}{4 b^{2}} c_{B}+\rho \frac{\sigma \eta}{a b}\right)\right\} .
$$

The first derivative of $y(t, x)$ with respect to $x$ at $x=0$ is given by (see the proof of Theorem 3)

$$
\lim _{x \downarrow 0} \frac{\partial y(t, x)}{\partial x}=-\frac{1}{2}(a(r(t)-\theta)-(a-b) Y(t)) .
$$

Solving the defining equation of $c_{B}$ to $Y(t)$ yields

$$
Y(t)=-\frac{\eta^{2}}{4 b^{2}} c_{B}-\frac{\eta^{2}}{2 b^{2}}-\rho \frac{\sigma \eta}{a b} .
$$

With this we have

$$
\lim _{x \downarrow 0} \frac{\partial y(t, x)}{\partial x}=-\frac{1}{2}\left(a(r(t)-\theta)+(a-b)\left(\frac{\eta^{2}}{4 b^{2}} c_{B}+\frac{\eta^{2}}{2 b^{2}}+\rho \frac{\sigma \eta}{a b}\right)\right) .
$$

Since we choose $c_{B}>-\frac{4 b^{2}}{\eta^{2}}\left(\frac{a}{a-b}(r(t)-\theta)+\frac{\eta^{2}}{2 b^{2}}+\rho \frac{\sigma \eta}{a b}\right)$, it holds

$$
\lim _{x \downarrow 0} \frac{\partial y(t, x)}{\partial x}>0 .
$$


Next, we consider the difference between $y(t, x)$ and $y_{\text {asymp }}$. For this, note

$$
\frac{B_{a}(x)}{x}>\frac{B_{b}(x)}{x}>\frac{B_{a+b}(x)}{x}>0, x>0 .
$$

With these inequalities, $c_{B}>1$, and $1-e^{-z x}>0$ for $x, z>0$, we can estimate the difference as follows:

$$
\begin{aligned}
y(t, x)-y_{\text {asymp }}= & -\frac{\sigma^{2}}{4 a^{2}} \frac{B_{a}(x)}{x}\left(c_{A}-\left(1-e^{-a x}\right)\right) \\
& -\frac{\eta^{2}}{4 b^{2}} \frac{B_{b}(x)}{x}\left(c_{B}-\left(1-e^{-b x}\right)\right)-\rho \frac{\sigma \eta}{a b} \frac{B_{a+b}(x)}{x} \\
> & -\frac{B_{a}(x)}{x}\left(\frac{\sigma^{2}}{4 a^{2}} c_{A}+\frac{\eta^{2}}{4 b^{2}} c_{B}\right)-\rho \frac{\sigma \eta}{a b} \frac{B_{a+b}(x)}{x} .
\end{aligned}
$$

For $\rho \leq 0$ we thus have

$$
y(t, x)-y_{\text {asymp }} \geq-\frac{B_{a}(x)}{x}\left(\frac{\sigma^{2}}{4 a^{2}} c_{A}+\frac{\eta^{2}}{4 b^{2}} c_{B}\right) .
$$

With the choice of $c_{A} \leq-\frac{a^{2} \eta^{2}}{b^{2} \sigma^{2}} c_{B}$, we obtain $y(t, x)-y_{\text {asymp }}>0$ for all $x>0$.

For $\rho>0$ we have

$$
\begin{aligned}
& -\frac{B_{a}(x)}{x}\left(\frac{\sigma^{2}}{4 a^{2}} c_{A}+\frac{\eta^{2}}{4 b^{2}} c_{B}\right)-\rho \frac{\sigma \eta}{a b} \frac{B_{a+b}(x)}{x}> \\
& -\frac{B_{a}(x)}{x}\left(\frac{\sigma^{2}}{4 a^{2}} c_{A}+\frac{\eta^{2}}{4 b^{2}} c_{B}+\rho \frac{\sigma \eta}{a b}\right) .
\end{aligned}
$$

Due to the choice of $c_{A} \leq-\frac{4 a^{2}}{\sigma^{2}}\left(\frac{\eta^{2}}{4 b^{2}} c_{B}+\rho \frac{\sigma \eta}{a b}\right)$, we obtain $y(t, x)-y_{\text {asymp }}>0$ for all $x>0$.

Consequently, $y(t, x)$ is greater than $y_{\text {asymp }}$ for the choice of $c_{A}$ and $c_{B}$.

Since $r(t)$ is greater than or equal to $y_{\text {asymp }}, \lim _{x \downarrow 0} \frac{\partial y(t, x)}{\partial x}$ is positive, $y(t, x)$ converges towards $y_{\text {asymp }}$ on the long end, and $y(t, x)$ lies above $y_{\text {asymp }}$, the yield curve has an odd number of local extreme values.

We write the first order condition for a local extreme value of $y(t, x)$ in case of $\rho \leq 0$ as

$$
\frac{\partial K_{c_{A}}(x)}{\partial x}+\frac{\partial R_{1}(x)}{\partial x}=-\frac{\partial K_{c_{B}}(x)}{\partial x}
$$

and in case of $\rho>0$ as

$$
\frac{\partial K_{c_{A}}(x)}{\partial x}=-\left(\frac{\partial K_{c_{B}}(x)}{\partial x}+\frac{\partial R_{1}(x)}{\partial x}\right) .
$$


$\frac{\partial K_{c_{A}}(x)}{\partial x}$ is strictly concave and $\frac{\partial K_{c_{B}}(x)}{\partial x}$ is strictly convex due to the choice of $c_{A} \leq-14$ and $c_{B}>1$ (see Lemma 2). $\frac{\partial R_{1}(x)}{\partial x}$ is strictly concave for $\rho<0$, it equals zero for $\rho=0$, and it is strictly convex for $\rho>0$. Consequently, $\frac{\partial K_{c_{A}}(x)}{\partial x}+\frac{\partial R_{1}(x)}{\partial x}$ and $-\frac{\partial K_{c_{B}}(x)}{\partial x}$ have at most two intersections for $\rho \leq 0$ according to Lemma 1. The same holds for $\frac{\partial K_{c_{A}}(x)}{\partial x}$ and $-\left(\frac{\partial K_{c_{B}}(x)}{\partial x}+\frac{\partial R_{1}(x)}{\partial x}\right)$ with $\rho>0$. Therefore, the derivative of $y(t, x)$ has at most two zeros and thus $y(t, x)$ at most two local extreme values.

Combined with the odd number of local extreme values that the yield curve must have, it follows that the yield curve is humped for the above choice of $r(t), c_{A}$, and $c_{B}$.

For $a>b$ we choose $c_{A}$ as

$$
c_{A} \geq 1
$$

and $c_{B}$ for $\rho \leq 0$ as

$$
c_{B}<\min \left\{-14 ;-\frac{4 b^{2}}{\eta^{2}}\left(\frac{a}{a-b}(r(t)-\theta)+\frac{\eta^{2}}{2 b^{2}}+\rho \frac{\sigma \eta}{a b}\right) ;-\frac{b^{2} \sigma^{2}}{a^{2} \eta^{2}} c_{A}\right\}
$$

and for $\rho>0$ as

$$
\begin{aligned}
c_{B}<\min \{- & 14 ;-\frac{4 b^{2}}{\eta^{2}}\left(\frac{a}{a-b}(r(t)-\theta)+\frac{\eta^{2}}{2 b^{2}}+\rho \frac{\sigma \eta}{a b}\right) ; \\
- & \left.\frac{4 b^{2}}{\eta^{2}}\left(\frac{\sigma^{2}}{4 a^{2}} c_{A}+\rho \frac{\sigma \eta}{a b}\right)\right\} .
\end{aligned}
$$

Analogously to $a<b$, it can be shown that $\lim _{x \downarrow 0} \frac{\partial y(t, x)}{\partial x}>0$ and $y(t, x)>y_{\text {asymp }}$ for the choice of $c_{A}$ and $c_{B}$. Due to the strict curvature of the derivative of $K_{c_{A}}(x), K_{c_{B}}(x)$, and $R_{1}(x), y(t, x)$ has a maximum of two local extreme values according to Lemma 1. Together with $r(t) \geq y_{\text {asymp }}, \quad \lim _{x \downarrow 0} \frac{\partial y(t, x)}{\partial x}>0, \quad \lim _{x \uparrow \infty} y(t, x)=y_{\text {asymp }}$ as well as $y(t, x)>y_{\text {asymp }}$, it follows that $y(t, x)$ is humped for the above choice of $r(t), c_{A}$, and $c_{B}$

(d) The case for dipped yield curves follows with obvious modifications. We now consider a short rate $r(t) \leq y_{\text {asymp }}$. For $a<b$ we choose $c_{B}$ as

$$
c_{B}<\min \left\{-14 ;-\frac{4 b^{2}}{\eta^{2}}\left(\frac{a}{a-b}(r(t)-\theta)+\frac{\eta^{2}}{2 b^{2}}+\rho \frac{\sigma \eta}{a b}\right)\right\}
$$

and $c_{A}$ for $\rho \leq 0$ as

$$
c_{A} \geq \max \left\{1 ;-\frac{4 a^{2}}{\sigma^{2}}\left(\frac{\eta^{2}}{4 b^{2}}\left(c_{B}-1\right)+\rho \frac{\sigma \eta}{a b}\right)+1\right\}
$$

and for $\rho>0$ as

$$
c_{A} \geq \max \left\{1 ;-\frac{a^{2} \eta^{2}}{b^{2} \sigma^{2}}\left(c_{B}-1\right)+1\right\} .
$$


For $a>b$ we choose $c_{A}$ as

$$
c_{A} \leq-14
$$

and $c_{B}$ for $\rho \leq 0$ as

$$
\begin{gathered}
c_{B}>\max \left\{1 ;-\frac{4 b^{2}}{\eta^{2}}\left(\frac{a}{a-b}(r(t)-\theta)+\frac{\eta^{2}}{2 b^{2}}+\rho \frac{\sigma \eta}{a b}\right) ;\right. \\
\left.-\frac{4 b^{2}}{\eta^{2}}\left(\frac{\sigma^{2}}{4 a^{2}}\left(c_{A}-1\right)+\rho \frac{\sigma \eta}{a b}\right)+1\right\}
\end{gathered}
$$

and for $\rho>0$

$$
\begin{aligned}
c_{B} & >\max \left\{1 ;-\frac{4 b^{2}}{\eta^{2}}\left(\frac{a}{a-b}(r(t)-\theta)+\frac{\eta^{2}}{2 b^{2}}+\rho \frac{\sigma \eta}{a b}\right) ;\right. \\
& \left.-\frac{b^{2} \sigma^{2}}{a^{2} \eta^{2}}\left(c_{A}-1\right)+1\right\} .
\end{aligned}
$$

It can be shown that we have

$$
\lim _{x \downarrow 0} \frac{\partial y(t, x)}{\partial x}<0 \text { and } y(t, x)<y_{\text {asymp }} .
$$

In particular, $y(t, x)$ has an odd number of local extreme values. Due to the strict curvature of the derivative of $K_{c_{A}}(x), K_{c_{B}}(x)$, and $R_{1}(x), y(t, x)$ has at most two local extreme values according to Lemma 1 . Together with

$$
r(t) \leq y_{\text {asymp }}, \lim _{x \downarrow 0} \frac{\partial y(t, x)}{\partial x}<0, \lim _{x \uparrow \infty} y(t, x)=y_{\text {asymp }}, \text { and } y(t, x)<y_{\text {asymp }},
$$

it follows that $y(t, x)$ is dipped for the above choice of $r(t), c_{A}$, and $c_{B}$.

Acknowledgements We would like to thank Ralf Werner for alerting us to the mistake in the published version of the original article.

Publisher's Note Springer Nature remains neutral with regard to jurisdictional claims in published maps and institutional affiliations. 